\title{
Correlation measurements of fission-fragment properties
}

\author{
S. Oberstedt ${ }^{1, a}$, T. Belgya ${ }^{2}$, R. Billnert ${ }^{3}$, R. Borcea ${ }^{1}$, D. Cano-Ott ${ }^{4}$, A. Göök ${ }^{5}$, \\ F.-J. Hambsch ${ }^{1}$, J. Karlsson ${ }^{3}$, Z. Kis ${ }^{2}$, T. Martinez ${ }^{4}$, A. Oberstedt ${ }^{3}$, L. Szentmiklosi ${ }^{2}$, \\ and K. Takác ${ }^{2}$ \\ 1 EC Joint Research Centre IRMM, Retieseweg 111, 2440 Geel, Belgium \\ 2 Department of Nuclear Research, IKI, 1525 Budapest, P.O. Box 77, Hungary \\ 3 School of Science and Technology, Örebro University, 70182 Örebro, Sweden \\ 4 CIEMAT, 28040 Madrid, Spain \\ 5 Institute for Nuclear Physics, Technical University, 64289 Darmstadt, Germany
}

\begin{abstract}
For the development of future nuclear fission applications and for a responsible handling of nuclear waste the a-priori assessment of the fission-fragments' heat production and toxicity is a fundamental necessity. The success of an indispensable modelling of the fission process strongly depends on a good understanding of the particular mechanism of scission, the mass fragmentation and partition of excitation energy. Experimental observables are fission-fragment properties like mass- and energy-distributions, and the prompt neutron as well as $\gamma$-ray multiplicities and emission spectra. The latter quantities should preferably be known as a function of fragment mass and excitation energy. Those data are highly demanded as published by the OECD-NEA in its high priority data request list. With the construction of the double (v, E) spectrometer VERDI we aim at measuring pre- and post-neutron masses directly and simultaneously to avoid prompt neutron corrections. From the simultaneous measurement of pre- and post-neutron fissionfragment data the prompt neutron multiplicity may then be inferred fully correlated with fragment mass yield and total kinetic energy. Using an ultra-fast fission event trigger spectral prompt fission $\gamma$-ray measurements may be performed. For that purpose recently developed lanthanum-halide detectors, with excellent timing characteristics, were coupled to the VERDI spectrometer allowing for a very good discrimination of fission $\gamma$-rays and prompt neutrons due to their different time-of-flight.
\end{abstract}

\section{Introduction}

In nuclear fission pairs of fission fragments with different mass and kinetic energy are produced [1]. This process is accompanied by prompt neutrons and $\gamma$-rays emitted from the highly excited fission fragments (FF). The knowledge of the FF yield-distribution, $\mathrm{Y}\left(\mathrm{A}, \mathrm{E}_{k}\right)$, as a function of mass (A) and kinetic energy $\left(\mathrm{E}_{k}\right)$ is a key input to fission models and important data for a better understanding the fission process as such. This information may be obtained by means of different techniques. By using a recoil mass separator [2] or measuring the time-of-flight together with the kinetic energy [3] single fission-fragment yields after prompt neutron emission may be obtained. Employing the double-energy (2E) method, fission-fragment characteristics prior to neutron emission, the so-called pre-neutron masses $\mathrm{A}^{*}$ and total kinetic energies, may be obtained, provided prompt neutron emission data are available for the iterative determination of the fragment mass [4,5]. It is just those latter data, which are available only for a few major actinides with sufficient accuracy.

\footnotetext{
a e-mail: stephan.oberstedt@ec.europa.eu
} permits unrestricted use, distribution, and reproduction in any noncommercial medium, provided the original work is properly cited. 
One way to avoid ambiguities introduced in the data analysis by the applied neutron corrections is to measure not only both kinetic energies, but simultaneously their velocities as well. Theoretically, from a double-velocity $(2 \mathrm{v})$ measurement both fragment masses before prompt-neutron evaporation may be obtained. The subsequent measurement of the fragment kinetic energies provides the information about the fission-fragment mass after prompt-neutron evaporation. Thus, the difference of pre- and post-neutron masses is just the number of evaporated prompt neutrons. In such a way, those important neutron data do not need to be introduced anymore in the data analysis. Instead, they may be directly deduced as a function of fragment mass and total kinetic energy, from which the excitation energy may be inferred. Such a double time-of-flight (TOF) and energy spectrometer had already been realized in the 1980s at the Institute Laue Langevin and was called Cosi-Fan-Tutte [3]. With an impressive mass resolution $\Delta \mathrm{A}$ well below 1 mass unit, it suffered from a very small geometrical efficiency of about $4 \times 10^{-5}$ [6]. Therefore, correlated FF-yield measurements were strongly affected by the non-colinearity of FF emission due to prompt neutron emission. That spectrometer does no longer exist.

Presently, a double (v, E) spectrometer is being built at the JRC-IRMM called VERDI [7], which aims at a FF mass resolution $\Delta \mathrm{A}<2$ in conjunction with a geometrical efficiency of at least $0.5 \%$, i.e. about 100 times more efficient than Cosi-Fan-Tutte. In order to achieve a high efficiency, the time pickup devices should be placed as close as possible to the target under investigation. Since this means, that such devices would have to be placed directly in the neutron beam, they have to be radiation resistant. Therefore, an artificial poly-crystalline chemical vapor deposited (pCVD) diamond film is considered as detector material to serve as fission event trigger.

Requests for new measurements on prompt $\gamma$-ray emission in the reactions ${ }^{235} \mathrm{U}\left(\mathrm{n}_{t h}, \mathrm{f}\right)$ and ${ }^{239} \mathrm{Pu}\left(\mathrm{n}_{t h}, \mathrm{f}\right)$ have been formulated and included in the Nuclear Data High Priority Request List of the Nuclear Energy Agency (NEA, Req. ID: H.3, H.4) [8]. However, a major difficulty in such measurements is, apart from the need to obtain a sufficient mass resolution for fission fragments, the clear suppression of background $\gamma$-rays induced by prompt fission neutrons in the $\gamma$-detector. A usual method here is to discriminate $\gamma$-rays and neutrons by their different time-of-flight. The quality of discrimination is strongly coupled to the timing resolution of the detector, which is usually not better than $5 \mathrm{~ns}$ for NaI detectors. A promising step towards better data might be achieved by using the recently developed cerium-doped lanthanum halide scintillation detectors, which have shown to provide a timing resolution better than 500 ps $[9,10]$ together with a more than $40 \%$ better energy resolution compared to $\mathrm{NaI}$, i.e. less than $4 \%$ (FWHM) compared to $6.5 \%$ at $662 \mathrm{keV}\left({ }^{137} \mathrm{Cs}\right)$ [11]. In conjunction with the ultra-fast fission trigger made from pCVD diamond excellent $\gamma$-ray/neutron discrimination may be expected.

In the following we report about a first correlation experiment, which has been performed at the Research Reseqrch Reactor in Budapest. The aim was to test the performance of a single TOF prototype of VERDI and the response of the new $\mathrm{LaCl}_{3}$ :Ce detectors operated in a high-flux neutron environment.

\section{Correlation measurements}

Measuring fission-fragments in coincidence with promptly emitted neutrons or $\gamma$-rays a detector system with high geometrical and detection efficiency is required. A fission-fragment detector with highest effciency is the double ionization chamber with Frisch-grids. The coincident measurement of promptly emitted $\gamma$-rays and/or neutrons was then possible with $\mathrm{NaI}$ detectors positioned at relatively large distance from the fission source. However, due to the above mentioned prompt-neutron correction to obtain mass and energy information fragment mass resolution is very much limited. Therefore, we contemplate single (v, E) or double-velocity measurements to avoid such corrections and to obtain higher mass resolution. The much less geometrical efficiency might be compensated by using stateof-the scintillation detectors with a much better timing resolution. They may be placed much closer to the fission source with at least the same discrimination power for fission $\gamma$-rays and prompt neutrons, hence, leading to an enhanced geometrical detection efficiency.

In the following two subsections the characterisation of the two components of the detector system, the time-of-flight (TOF) spectrometer VERDI and the very fast scintillation detectors made of 
cerium-doped lanthanum-chloride $\left(\mathrm{LaCl}_{3}: \mathrm{Ce}\right)$, is briefly recalled before first results of an in-situ test are presented.

\subsection{The time-of-flight (TOF) spectrometer VERDI}

The VERDI spectrometer provides a flight-path length of $50 \mathrm{~cm}$. The energy of the fission fragments is measured with large area silicon (PIPS) detecors. In total 19 detectors will be placed in each TOF section to achieve the contemplated geometrical efficiency of $0.5 \%$. The time-of-flight is measured between a start-detector closely placed at the fission sample and a PIPS detector. The start detector is made from artifical poly-crystalline diamond (pCVDD).

In nuclear physics diamond detectors are used mainly in high-energy experiments as beam monitors and tracking devices, replacing traditionally employed silicon detectors, because they survive in high radiation environments, have low leakage current and do not need cooling [12-15]. In particular the timing properties of artificial diamonds are remarkable and an intrinsic timing resolution better than $30 \mathrm{ps}$ has been achieved for a mono-energetic ${ }^{52} \mathrm{Cr}$-beam at incident energy of $650 \mathrm{MeV} / \mathrm{u}[16]$. In view of the properties of this surprising material, it was tempting to see whether a similar timing resolution may be obtained with low energy fission fragments at energies typically between 0.5 and $2.0 \mathrm{MeV} / \mathrm{u}$.

Since this detector is not yet transparent for FF we restrict ourselves to discuss a single TOF configuration.

First, the diamond detectors were irradiated with $\beta$-particles (the so-called priming) before exposed to highly ionizing particles like fission fragments $[17,18]$. Then, we monitored the pulse-height signal stability up to more than $10^{9}$ fission fragments from a ${ }^{252} \mathrm{Cf}$ source together with the corresponding number of $\alpha$-particles and fission neutrons for which no signal degradation was observed. Next, we measured the fission-fragment time-of-flight with a symmetric set-up consisting of two identical pCVDDDs. The intrinsic timing resolution was then inferred by means of a Monte-Carlo simulation based on published post-neutron data from the reaction ${ }^{252} \mathrm{Cf}(\mathrm{SF})$. The experiment has been performed two-fold: first, using standard analogue electronics and second, using a digital oscilloscope with a bandwidth of $1 \mathrm{GHz}$. From various simulations an intrinsic timing resolution $\sigma_{\text {int }}<300 \mathrm{ps}$ for the analogue and $\sigma_{\text {int }} \approx 150$ ps for the digital measurement was obtained. The spectrum obtained with digital electroniques is dipicted in Fig. 1 together with the results from the Monte-Carlo simulations. The timing resolution is comparable with the better micro-channel plate detectors, but diamond detectors are much easier to handle and to operate.

In a next step the TOF spectrum of fission fragments from spontaneous fission of ${ }^{252} \mathrm{Cf}$ was measured with a configuration consisting of one pCVDDD and a small PIPS detector of size $25 \mathrm{~mm}^{2}$

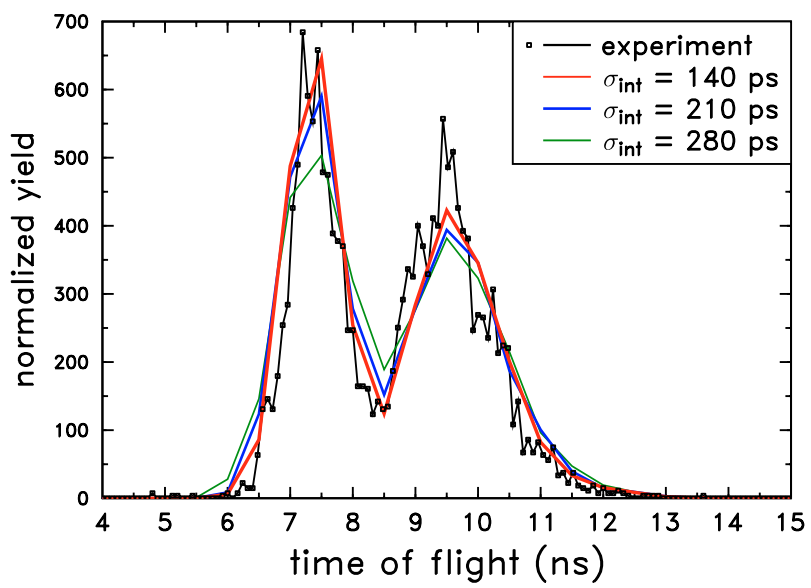

Fig. 1. Fission fragment time-of-flight for the reaction ${ }^{252} \mathrm{Cf}(\mathrm{SF})$ taken with a waveform digitizer with a bandwidth of $1 \mathrm{GHz}$. 


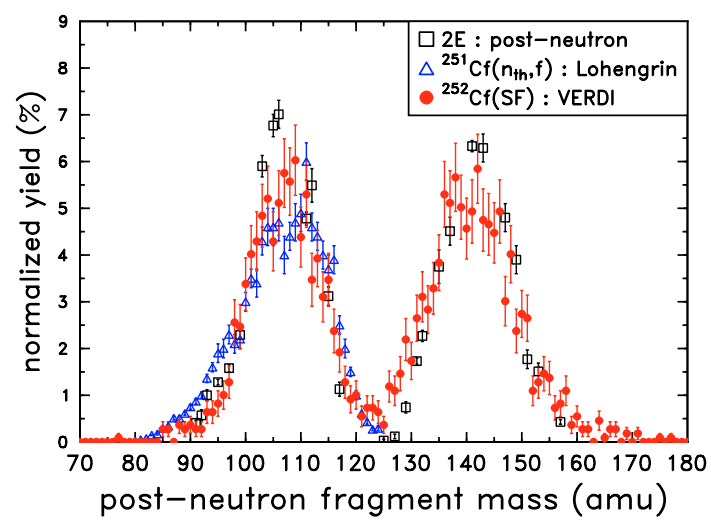

Fig. 2. Experimental fission-fragment mass distribution from the reaction ${ }^{252} \mathrm{Cf}(\mathrm{SF})$ obtained from a TOF measurement with a pCVDDD as fission trigger and a $25 \mathrm{~mm}^{2}$ silicon-type energy detector, compared to results from Refs. [19-21].

separated by only $33 \mathrm{~cm}$. The obtained timing resolution was around $500 \mathrm{ps}$ with conventional analogue electronics. The resulting fragment mass-distribution is shown in Fig. 2 as red dots and compared with several data existing in literature [19-21]. The comparison with data from a $2 \mathrm{E}$ measurement shows good agreement and suggests an improved mass resolution already at the reduced flight-path length. In the region of symmetric masses and at the distribution wings random triggers with $\alpha$-particles are visible. From this test measurement a mass resolution $\Delta \mathrm{A}<3$ may be expected with analogue electronics.

\subsection{Lanthanum-halide detctors for prompt-fission $\gamma$-ray measurements}

In preparation of prompt fission $\gamma$-ray measurements two $1.5^{\prime \prime} \times 1.5^{\prime \prime}$ lanthanum-cloride detectors from the company SCIONIX[22] have been characterized, which is described in detail elsewhere[24]. They were found to have an intrinsic timing resolution of about $440 \mathrm{ps}$, measured with a ${ }^{60} \mathrm{Co}$ source. The energy resolution was determined for $\gamma$-energies from 81 to $6919 \mathrm{keV}$, exhibiting more or less the expected $E^{-1 / 2}$ behaviour. For $662 \mathrm{keV}\left({ }^{137} \mathrm{Cs}\right)$ the obtained energy resolution was around $4 \%$ (FWHM), in agreement with values provided by the manufacturer[22]. Together with a dynamical range up to more than $11 \mathrm{MeV}$, these detectors showed a good linearity with residuals far below $1 \%$. The full intrinsic peak efficiency was observed to be $53 \%$ better than the one for NaI:Tl detectors of the same size[25].

\section{First in-situ test with reactor neutrons}

The experimental set-up was installed at the cold neutron beam of the IKI Research Reaktor in Budapest. The neutron flux at the entrance window of the VERDI spectrometer was $5 \times 10^{7}$ neutrons $/ \mathrm{s} / \mathrm{cm}^{2}$.

During the experiment VERDI was operated in a single $(\mathrm{v}, \mathrm{E})$ configuration. Ten $450 \mathrm{~mm}^{2}$ large PIPS detectors are placed at a distance of $50 \mathrm{~cm}$ from the fission source. As fissile target a $113 \mu \mathrm{g}$ ${ }^{235} \mathrm{U}$, mounted on a $34 \mu \mathrm{g} / \mathrm{cm}^{2}$ thick polyimide backing, was used and placed directly on the diamond detector. On top of the fissile sample a ${ }^{6} \mathrm{Li}$ sample was placed to use the ${ }^{6} \mathrm{Li}(\mathrm{n}, \mathrm{t}) \alpha$ reaction for time calibration. The shaped pulse-height $(\mathrm{PH})$ signals of the PIPS detectors were fed into an analogue signal-router before fed into a single ADC. In total five words, one PH information and three TAC (FF TOF) values and a bit-pattern for detector identification, were stored by the DAQ in listmode for later off-line treatment. 


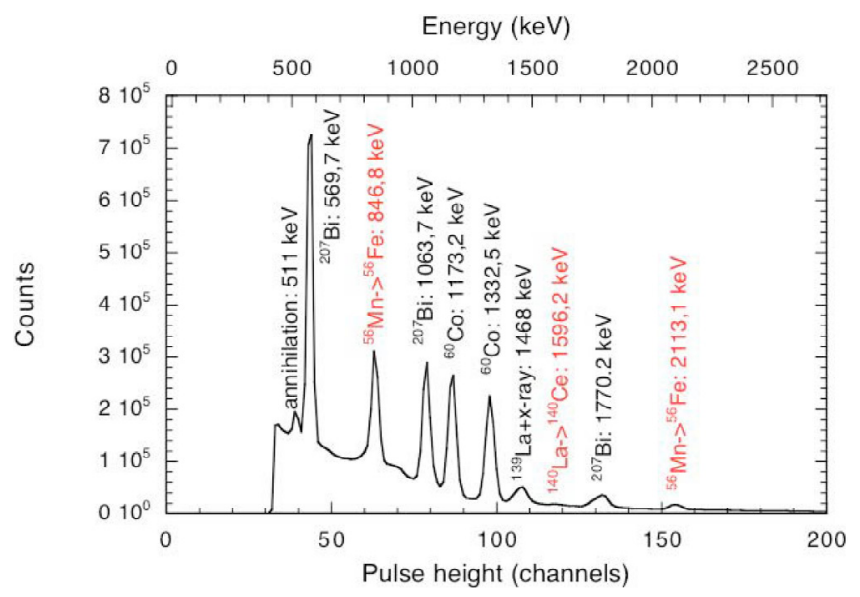

Fig. 3. Pulse height calibration spectrum of the $3^{\prime \prime} \times 3^{\prime \prime} \mathrm{LaCl}_{3}$ :Ce detector. The different colours of the energy assignments refer to $\gamma$-peaks from the calibration sources, ${ }^{60} \mathrm{Co}$ and ${ }^{207} \mathrm{Bi}$, and from activation in the detector and its environment, respectively.

Three $\mathrm{LaCl}_{3}$ detecors are placed outside of the VERDI spectrometer at a distance of $30 \mathrm{~cm}$ from the fission source. One detector was of size $3^{\prime \prime} \times 3^{\prime \prime}$ [23] and the others of size $1.5^{\prime \prime} \times 1.5^{\prime \prime}$ [22]. The properties of the larger $\mathrm{LaCl}_{3}$ :Ce detector were not fully known at the time of the experiment, its efficiency, however, could be expected to be more than three times higher compared to the smaller detectors around $1 \mathrm{MeV}[26]$, mostly for geometrical reasons.

Additionally, a $2^{\prime \prime} \times 2^{\prime \prime}$ large $\mathrm{LaBr}_{3}$ [23] detector was placed for testing in a neutron field. The detector was delivered just before the start of the experiment and was, thus, not characterized yet. Nevertheless, from previous studies it is known that these detectors are even better than $\mathrm{LaCl}_{3}: \mathrm{Ce}$ detectors in terms of both energy and timing resolution, with about $2.8 \%$ (FWHM) at $662 \mathrm{keV}$ and coincidence resolving times of $<300 \mathrm{ps}$ [10].

The signals from the four $\gamma$-detectors were fed into constant fraction discriminators (CFD) and further into the same time-to-analog converter (TAC) of range $1 \mu \mathrm{s}$. They were giving the start signal, while the signals from the diamond detector, with an appropriate delay, provided the stop signal of the coincidence. For all four scintillation detectors the pulse height was stored in listmode, together with three pulse shape discrimination signals for the $\mathrm{LaCl}_{3}$ :Ce detectors only, as well as the TAC signal in a separate DAQ.

Prior to and right after the beamtime the detectors were energy and efficiency calibrated using different radioactive sources. Figure 3 shows the calibration spectrum of the large $\mathrm{LaCl}_{3}: \mathrm{Ce}_{\text {detector}}$, taken with a ${ }^{60} \mathrm{Co}$ and a ${ }^{207} \mathrm{Bi}$ source after neutron irradiation of the target. The $\gamma$-peaks are assigned to the corresponding source and energy, the assignments in a lighter colour, however, belong to peaks that were not observed before the neutron beam was opened. They were identified to correspond to activation and successive decay of atoms in the crystal by the reaction ${ }^{139} \mathrm{La}\left(\mathrm{n}_{t h}, \gamma\right)$ and induced by fission neutrons through the reaction ${ }^{56} \mathrm{Fe}(\mathrm{n}, \mathrm{p}){ }^{56} \mathrm{Mn}$ in the wall of the vacuum chamber of VERDI, respectively. Apart from that, we noticed that the conversion from pulse height to energy practically did not change over the period of 10 days of experiment. Moreover, from the counts under the $1596.2 \mathrm{keV}$ peak, the known volume and efficiency of the detector we could estimate the thermal neutron flux impinging on the detectors to be less than $0.2 / \mathrm{cm}^{2} / \mathrm{s}$.

In Fig. 4 the VERDI spectrometer is shown together with the four $\gamma$-ray detectors.

In Fig. 5 a fission-fragment TOF-spectrum is shown together with two Monte-Carlo simulations each with a differentvalue for the timing resolution of the spectrometer. Due to problems with the priming source the discriminatorthreshold had to be set relatively low. This increased the number of accidential coincidences between one fission-fragment and an $\alpha$-particle from the ${ }^{6} \mathrm{Li}(\mathrm{n}, \mathrm{t}) \alpha$ reaction or the natural activity of the ${ }^{234} \mathrm{U}$ present in the ${ }^{235} \mathrm{U}$ target, which explaines the tails visible on the right side of the spectrum peaks shown in Fig. 5. From the Monte-Carlo simulations a timing resolution 


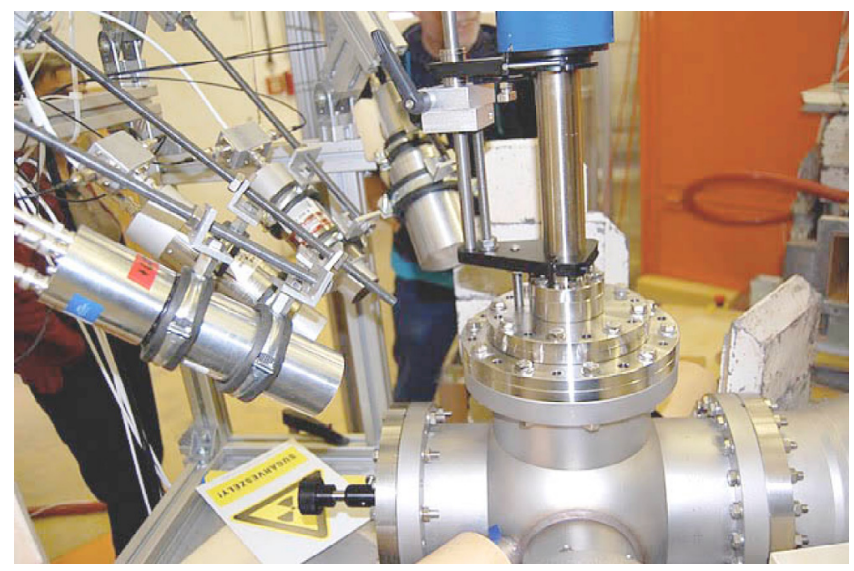

Fig. 4. Photograph of the experimental setup with four lanthanum halide scintillation detectors (upper left part) and the fission fragment spectrometer VERDI. The neutron beam enters from the lower edge.

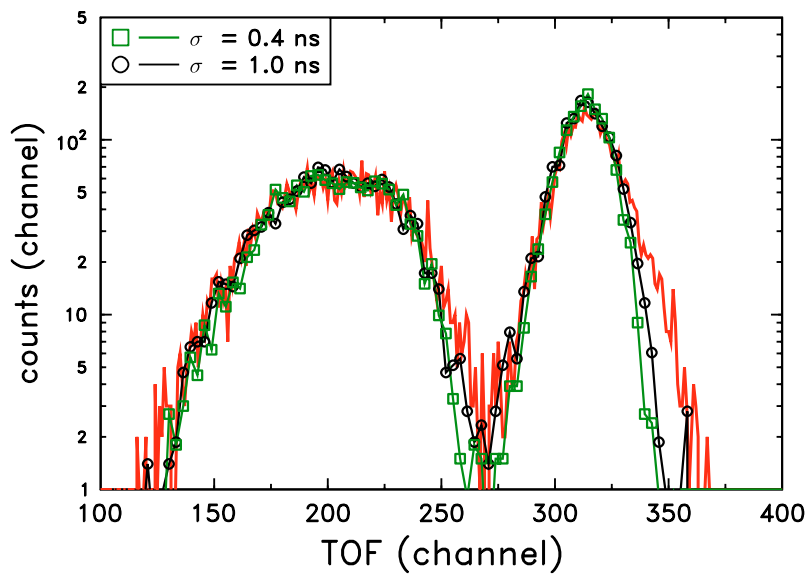

Fig. 5. Raw TOF spectrum obtained from the reaction ${ }^{235} \mathrm{U}\left(\mathrm{n}_{t h}, \mathrm{f}\right)$ together with Monte-Carlo simulations made for two different timing resolutions (see text).

of VERDI of about $400 \mathrm{ps}$ is deducted. The off-line analysis of all 10 detectors to obtain the postneutron mass distribution, which permits to gate out a major part of the accidential coincidences, is still ongoing.

The prompt fission $\gamma$-ray spectra were obtained from the measured time spectra. For each detector the data treatment was carried out as follows:

- first a selection was made on the pulse shape signal (where available) in order to discriminate the $\gamma$-rays from other incident particles, including $\alpha$-particles from the intrinsic activity of the crystals

- then the dependence of the TAC-signal from pulse height was determined and corrected for

- different windows were set in order to distinguish events from different $\gamma$-ray producing reactions, i.e. prompt fission as well as thermal and fast neutron induced process such as $\left(n_{t h}, \gamma\right),\left(n, n^{\prime}\right)$ and $(n, p)$

An example for the results of this procedure is shown in the upper part of Fig. 6. There, the rectangular regions are indicating in which TAC-signal regime the different processes were expected. During data analysis, however, these windows were chosen as large as possible. For each of them pulse height spectra were generated and energy calibrated and, the processes were verified by identifying the characteristic $\gamma$-spectra. The thermal-neutron induced $\gamma$-rays (including the ones from the activation of VERDI) 

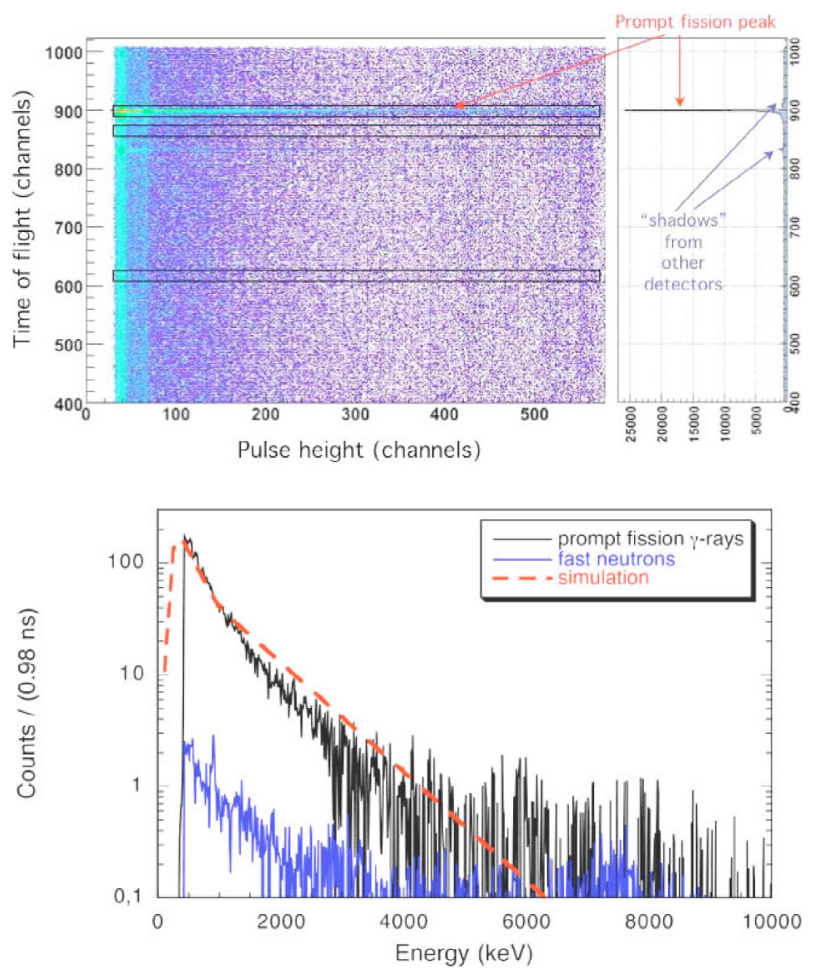

Fig. 6. Upper part: Two-dimensional representation of $\gamma$-rays detected with the large $\mathrm{LaCl}_{3}$ :Ce detector by their TAC-signal versus pulse height. To the right a projection on the TAC-axis is shown, indicating the prompt fission $\gamma$-rays together with some corresponding events from other detectors. The three rectangles denote areas, where prompt fission $\gamma$-rays (upper) and $\gamma$-rays from fast neutron (middle) and thermal neutron (lower) induced reactions are observed; lower part: Normalized, energy-calibrated and background-subtracted spectra for the 3 in. $\times 3$ in. $\mathrm{LaCl}_{3}$ :Ce detector from both inelastic scattering of fast neutrons and prompt decay of fission products together with the result of simulations.

were not correlated with fission fragments and thus independent from time. They were considered as background, normalized per TAC-channel and subtracted from the other spectra. Also the $\gamma$-rays from inelastic neutron scattering were subtracted from the prompt $\gamma$-rays, which results in the energy distribution of prompt fission $\gamma$-rays. The lower part of Fig. 6 shows normalized, energy-calibrated and background-subtracted spectra for the large $\mathrm{LaCl}_{3}$ :Ce detector from both inelastic scattering of fast neutrons and prompt decay of fission products. To guide the eye, the result of a simulation [27] is included and compared to the experimental fission $\gamma$-ray spectrum, which is described by

$$
N(E)=\left\{\begin{array}{lr}
38.13(E-0.085) e^{1.648 E} & E<0.3 \mathrm{MeV} \\
26.8 e^{-2.30 E} & 0.3<E<1.0 \mathrm{MeV} \\
8.0 e^{-1.10 E} & 1.0<E<8.0 \mathrm{MeV}
\end{array}\right.
$$

as obtained from previous measurements [28]. Although the experimental spectrum is not corrected for efficiency yet and only a part of the taken data is analyzed so far, the good agreement is promising. What remains to be done is the analysis of all acquired data as well as determining the response functions for all detectors and applying them to the spectra. 


\section{Summary and conclusions}

In this paper we have presented a set-up for measuring correlated fission-fragment characteristics. The instrument consists of very fast timing and $\gamma$-ray energy-resolving lanthanum halide detectors coupled to the fission-fragment time-of-flight spectrometer VERDI. The time pick-up detector is made from artificial diamond. The intrinsic timing resolution of pCVDD detectors determined with standard analogue electronics is well below $300 \mathrm{ps}$. The use of state-of-the-art low-noise broadband electronics may bring the resolution down to well below 150 ps as suggested by analysing signals taken with a $1 \mathrm{GHz}$ waveform digitizer. Radiation hardness was verified for typical fission fragment, $\alpha$-particle and neutron doses. A first in-situ test of the single time-of-flight prototype of the VERDI spectrometer demonstrated the suitability of the spectrometer components. Today a timing resolution of VERDI as good as $400 \mathrm{ps}$ was deducted from Monte-Carlo simulations, which may be translated into a mass resolution $\Delta \mathrm{A} \approx 3$.

First and preliminary results from the measurement of prompt $\gamma$-rays from the reaction ${ }^{235} U\left(n_{t h}, f\right)$ were presented. Despite the early stage of data analysis the comparison with a simulation of the energy distribution appears encouraging. This makes us optimistic to provide eventually new and more precise data to resolve present deficiencies in $\gamma$-ray production data in evaluated nuclear data files. The employment of fast detectors like lanthanum halide detectors in conjunction with pCVD diamond detectors will account for that.

We may conclude, that the double time-of-flight spectrometer VERDI coupled to a set of lanthanumhalide detectors is a promising instrument for correlated measurements of fission fragment characteristics with a relatively high geometrical efficiency. The instrument will be best suited for use at medium- and high-flux neutron facilities.

This work was supported by EFNUDAT (agreement number 31027) and NAP VENEUS05 (agreement number OMFB 00184/2006).

\section{References}

1. The Nuclear fission process, editor C. Wagemans, CRC Press, ISBN 0-8493-5434-X (1991).

2. E. Moll, H. Schrader, G. Siegert, H. Hammers, M. Asghar, J. P. Bocquet, P. Armbruster, H. Ewald and H. Wollnik, Kerntechnik 8, (1977) 374.

3. A. Oed, P. Geltenbort, R. Brissot, F. Gönnenwein, P. Perrin, E. Aker, D. Engelhardt, Nucl. Inst. Meth. in Phys. Res. 219, (1984) 569.

4. C. Budtz-Jørgensen, H.-H. Knitter, Ch. Straede, F.-J. Hambsch, R. Vogt, Nucl. Inst. Meth. A 258, (1987) 209.

5. F. Vivès, F.-J. Hambsch, H. Bax, S. Oberstedt, Nucl. Phys. A 662, (2000) 63.

6. N. Boucheneb, M. Ashgar, G. Barreau, T. P. Doan, B. Leroux, A. Sicre, P. Geltenbort and A. Oed, Nucl. Phys. A 535, (1991) 77.

7. http://www.fysikersamfundet.se/kf/arsmoten/2009/talks/slides_oberstedt_stephan.pdf

8. http://www.nea.fr/html/dbdata/hprl/hprlview.pl?ID=421 and http://www.nea.fr/html/dbdata/hprl/ hprlview.pl?ID=422.

9. K. S. Shah, J. Glodo, M. Klugerman, L. Cirignano, W. W. Moses, S. E. Derenzo, M. J Weber, Nucl. Instr. Meth. A 505, (2003) 76.

10. A. Iltis, M. R. Mayhugh, P. Menge, C. M. Rozsa, O. Selles, V. Solovyev, Nucl. Instr. Meth. A 563, (2006) 359.

11. B. D. Milbrath, B. J. Choate, J. E. Fast, W. K. Hensley, R. T. Kouzes, J. E. Schweppe, Nucl. Instr. Meth. A 572, (2007) 774.

12. RD 42 Collaboration, P. Weilhammer et al., Nucl. Inst. Meth. A 409, (1998) 264.

13. The RD42 collaboration, D. Meier et al., Nuc. Inst. Meth. A 426, (1999) 173.

14. E. Berdermann, K. Blasche, P. Moritz, H. Stelzer, "Diamond Detectors for Heavy-Ion Measurements", XXXVI Int. Winter Meeting on Nuclear Physics, Bormio 1998. 
15. E. Berdermann, K. Blasche, P. Moritz, H. Stelzer, B. Voss, F. Zeytouni, Nucl. Phys. B (Proc. Suppl.) 78, (1999) 533-539.

16. E. Berdermann, K. Blasche, P. Moritz, H. Stelzer, B. Voss, Diamond and Related Materials 10, (2001) 1770-1777.

17. M. Marinelli, E. Milani, A. Paoletti, A. Tucciarone, G. Verona Rinati, M. Angelone, M. Pillon, Nucl. Inst. Meth. A 476, (2002) 701-705.

18. S. Oberstedt, C. C. Negoita, T. Atzitzoglu, Scientific Report of the Neutron Physics Unit 2006, EUR Report 23039 EN, Eds. S. Oberstedt, P. Rullhusen, ISBN 978-92-79-05365-8 (2007) 95.

19. E. Birgersson, S. Oberstedt, A. Oberstedt, F.-J. Hambsch, D. Rochman, I. Tsekhanovich, S. Raman, Nucl. Phys. A 791, (2007) 1-23.

20. F.-J. Hambsch and S. Oberstedt, Nucl. Phys. A 617, (1997) 347-355.

21. F.-J. Hambsch (2004), private communication.

22. SCIONIX Holland bv, P.O. Box 143, 3980 CC Bunnik, The Netherlands.

23. Saint-Gobain Crystals, 17900 Great Lakes Pkwy, Hiram, OH 44234-9681, USA.

24. A. Oberstedt, R. Billnert, J. Karlsson, S. Oberstedt, W. Geerts, and F.-J. Hambsch, in: A. Chatillon, H. Faust, G. Fioni, and D. Goutte, H. Goutte (Eds.), Fourth Int. Workshop on Nucl. Fission and Fission-Product Spectroscopy, AIP Conf. Proc., vol. 1175, 2009, p. 257.

25. G. Gilmore, Practical Gamma-ray Spectroscopy, John Wiley \& Sons, ISBN 978-0-470-86196-7 (2008).

26. R. Nicolini, F. Camera, N. Blasi, S. Brambilla, R. Bassini, C. Boiano, A. Bracco, F. C. L. Crespi, O. Wieland, G. Benzoni, S. Leoni, B. Million, D. Montanati, A. Zalite, Nucl. Instr. Meth. A 582 (2007) 554.

27. J. M. Verbeke, C. Hagmann, D. Wright, UCRL-AR-228518, Lawrence Livermore National Laboratory (2009).

28. F. C. Maienschein, R. W. Peelle, T. A. Love, Neutron Phys. Ann. Prog. Rep. for Sept. 1, 1958, ORNL-2609, Oak Ridge National Laboratory (1958). 